\title{
Disposición a la investigación científica en estudiantes de ciencias de la salud
}

Eduard Antonio Maury-Sintjago¹, Estefanía Valenzuela-Figueroa', Massiel Henríquez-Riquelme', Alejandra RodríguezFernández ${ }^{1}$

\section{RESUMEN}

Objetivo: Determinar la disposición que tienen estudiantes chilenos de ciencias de la salud a la investigación científica. Materiales y métodos: Estudio observacional, descriptivo, de corte transversal, prospectivo. La muestra estuvo conformada por 246 estudiantes de ciencias de la salud. Se aplicó un cuestionario de actitudes frente a la investigación que contenía 22 ítems (a Cronbach =0,895) en escala tipo Likert ( 1 al 5). Se aplicó estadística descriptiva e inferencial. La significancia se estableció a un p:0,05.

Resultados: El puntaje medio del cuestionario fue de $2,7 \pm 1,01$, indicando una baja disposición por parte de estudiantes a la investigación, los ítems con puntajes más bajos fueron los asociados al análisis estadístico y búsqueda de artículos científicos. La carrera con mayor índice de disposición fue Enfermería, mientras que Terapia Ocupacional y Fonoaudiología tuvieron la menor disposición.

Conclusiones: Es necesario fomentar en el estudiantado el reconocimiento de la importancia de basamento científico en el abordaje de situaciones de salud.

Palabras claves: Actitudes; investigación; estudiantes del área de la salud (Fuente: DeCS BIREME).

\section{Willingness for scientific research in health science students}

\section{ABSTRACT}

Objective: To determine the willingness for scientific research in Chilean health science students.

Materials and methods: An observational, descriptive, cross-sectional, prospective study. The sample consisted of 246 health science students. A 22-item questionnaire on attitudes to research (a Cronbach $=0.895$ ) was conducted using a 5-point Likert scale (1 to 5). Descriptive and inferential statistics were applied. Significance was established at $p=0.05$. Results: The questionnaire mean score was $2.7 \pm 1.01$, which indicated low willingness for scientific research in health science students. The items with the lowest scores were associated with statistical analysis and scientific literature search. The academic program with the highest willingness index was nursing, while occupational therapy and phonoaudiology had the lowest willingness indexes.

Conclusions: It is necessary to promote in students the importance of scientific grounding to face health situations.

Keywords: Attitudes; research; students, health occupations (Source: MeSH NLM).

1. Universidad del Bío-Bío, Facultad de Ciencias de la Salud y de los Alimentos, Departamento de Nutrición y Salud Pública. Chillán, Chile. 


\section{INTRODUCCIÓN}

La práctica formativa en el área de las ciencias de la salud debe hacer frente al desafío de generar en los estudiantes un proceso reflexivo que les estimule al cuestionamiento constante de los fenómenos. Por tanto, se debe favorecer en los estudiantes una actitud positiva para la generación del conocimiento y la ciencia ${ }^{(1)}$.

Chile es uno de los países de la región con mayor número de publicaciones científicas en el área de la salud y ocupa un lugar importante en producción científica estudiantil en Latinoamérica ${ }^{(2,3)}$. Sin embargo, la producción científica estudiantil se concentra fuertemente en estudiantes de medicina por encima de las otras áreas de las ciencias de la salud, por lo que se hace menester incrementar la participación de otros estudiantes.

Son escasos los estudios que buscan identificar la disposición que tienen los estudiantes de ciencias de la salud hacia la investigación científica. No obstante, la poca y empírica información existente da cuenta de una alta variabilidad de disposición hacia la investigación por parte de estudiantes. Silva et al. (2013), en su estudio con estudiantes panameños muestra de un deficiente conocimiento y una actitud regular acerca de la investigación ${ }^{(4)}$. Estos resultados difieren con lo encontrado por Plazas et al. (2013), en investigación con estudiantes colombianos donde encontró que más del $80 \%$ de los encuestados tienen una actitud positiva hacia la investigación (1). Concordando con lo encontrado por Pelcastre (2015), en su estudio con estudiantes mexicanos ${ }^{(5)}$.

Dado que existe poca evidencia sólida y alta variabilidad entre estudiantes preuniversitarios, universitarios y entre estudiantes de varias áreas del saber, esta investigación tiene como objetivo determinar la disposición a la investigación científica en estudiantes chilenos de ciencias de la salud.

\section{MATERIALES Y MÉTODOS}

Se realizó un estudio observacional, analítico de corte transversal prospectivo. La muestra estuvo constituida por 246 estudiantes de algunas carreras de ciencias de la salud: Nutrición y Dietética, Kinesiología, Enfermería, Terapia Ocupacional y Fonoaudiología, de universidades acreditadas y localizadas en la zona urbana de la ciudad de Santiago de Chile.

En todas las carreras estudiadas había alumnos de distinto nivel académico (primer año a cuarto año); sin embargo, no participaron los estudiantes de quinto año puesto que éstos se encuentran distribuidos a tiempo completo en sus centros de práctica. Todos los participantes debían cumplir como criterio de inclusión ser estudiantes regulares de la universidad y completar la totalidad de la encuesta. Aquellas encuestas con carencia de algún dato fueron excluidas.

El instrumento utilizado contenía dos secciones: a y b. La sección "a" contenía información general, como: sexo, edad, carrera, nivel académico, experiencia en investigación. Por su parte, la sección "b" consistía en un cuestionado traducido y validado por Silva et al. (2013), en estudiantes de medicina panameños ${ }^{(4)}$. El cuestionario, para determinar la disposición que tienen los estudiantes frente a la investigación científica, estaba constituido por 22 ítems contenidos en diferentes dimensiones relacionadas a la actitud frente a la investigación científica, tales como la lectura crítica de artículos científicos, preparación de protocolos de investigación, participación de comités científicos, preparación de manuscritos, análisis estadístico y divulgación de resultados en eventos científicos. El cuestionario tiene una escala tipo Likert que va desde 1 a 5 , siendo 1 la peor disposición y 5 la mejor, se consideró como una actitud positiva aquel promedio final $\geq 3,5$. Finalmente, para corroborar la consistencia del cuestionario, se aplicó una prueba piloto entre los estudiantes obteniendo un alfa de Cronbach de 0,895.

Dada la naturaleza de la investigación los datos fueron expresados en frecuencias absolutas y relativas, promedios y desviación estándar. La distribución normal de las variables continúas fue verificada con la prueba de Shapiro-Wilks. Para comparar las frecuencias relativas entre grupos se utilizó la prueba de $\mathrm{chi}^{2}$ y para comparar los datos continuos se utilizó la prueba de ANOVA con posthoc de Tukey. La significancia estadística se estableció en un valor $p<0,05$. Para el análisis de los datos se utilizó el programa estadístico SPSS V 21.0.

A todos los participantes se les explicó mediante una charla informativa la finalidad del estudio; asimismo, todos debieron firmar el consentimiento informado pudiendo abstenerse de participar en la investigación. Se respetaron los principios bioéticos y el anonimato de los participantes y de la institución.

\section{RESULTADOS}

Se aplicaron 246 encuestas distribuidos de la siguiente manera, Nutrición y Dietética $(n=34)$, Kinesiología $(n=44)$, Enfermería $(n=132)$, Fonoaudiología $(n=19)$ y Terapia Ocupacional $(n=17)$. Por tanto el número de participantes $(53,7 \%)$ pertenecían a la carrera de Enfermería seguida de Kinesiología $(17,9 \%)$ mientras que la carrera con menos participantes fue Terapia Ocupacional con tan solo un $6,9 \%$. La mayoría de la muestra pertenecía al sexo femenino $(61,8 \%)$. Con respecto al nivel cursado, se recolectó mayor número de participantes en primer año (31,7\%). Finalmente, solo el $4,1 \%$ que equivale a 10 personas de un total de 246, mencionó tener experiencia en investigación (Tabla 1). 
Tabla 1. Características generales de los participantes del estudio

\begin{tabular}{|lcc|}
\hline \multicolumn{1}{|c}{ Variable } & N & $\%$ \\
\hline Sexo & & \\
Femenino & 152 & 61,8 \\
\hline Masculino & 94 & 38,2 \\
\hline Carreras & & \\
\hline Nutrición y Dietética & 34 & 13,8 \\
Kinesiología & 44 & 17,9 \\
\hline Enfermería & 132 & 53,7 \\
\hline Terapia Ocupacional & 17 & 6,9 \\
\hline Fonoaudiología & 19 & 7,7 \\
\hline Nivel académico & & \\
Primer año & 78 & 31,7 \\
\hline Segundo año & 63 & 25,6 \\
\hline Tercer año & 55 & 22,4 \\
\hline Cuarto año & 50 & 20,3 \\
\hline Experiencia en investigación & & \\
\hline Sí & 10 & 4,1 \\
\hline No & 236 & 95,9 \\
\hline
\end{tabular}

En la tabla 2, se presentan el promedio y desviación estándar de las puntuaciones por cada ítem recogido en el cuestionario de disposición frente a la investigación.

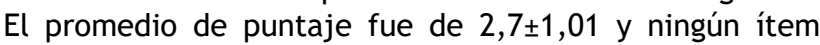
tuvo una ponderación media superior a 3,5. Los ítems de disposición que tuvieron mayor puntuación fueron “Otorgar créditos académicos a los estudiantes que realicen investigaciones, publicaciones y presentaciones científicas" y "Realizar investigación científica durante

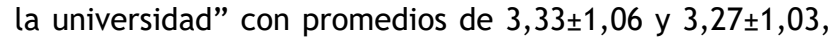
respectivamente. Por su parte los ítems con menor puntaje fueron "Utilizar programas estadísticos para realizar análisis estadísticos” y “Búsqueda de artículos científicos y revisión bibliográfica de póster" con $1,99 \pm 0,85$ y 2,05 $\pm 0,91$ de promedios, respectivamente.

Tabla 2. Puntuación asignada a cada ítem del cuestionario de disposición a la investigación científica en los sujetos de estudio

\begin{tabular}{lc}
\multicolumn{1}{c}{ Variable } & $\%$ \\
\hline Utilizar programas estadísticos para realizar análisis estadísticos & $1,99 \pm 0,85$ \\
Búsqueda de artículos científicos y revisión bibliográfica de póster & $2,05 \pm 0,91$ \\
Enviar, corregir y tramitar un artículo científico hasta que sea publicado en una revista científica & $2,13 \pm 0,87$ \\
Lectura crítica de un artículo científico y aplicación de la medicina basada en evidencia & $2,24 \pm 0,83$ \\
Implementar una rotación durante los semestres clínicos por un centro especializado en investigación & $2,20 \pm 0,88$ \\
Redactar un protocolo de investigación & $2,23 \pm 0,89$ \\
Presentar una investigación científica en un congreso o seminario en formato de charla (oral) & $2,18 \pm 0,95$ \\
Redactar un artículo científico (incluyendo la elaboración de gráficas, tablas, etc.) & $2,29 \pm 0,86$ \\
Presentar una investigación científica en un congreso o seminario en formato & $2,32 \pm 0,87$ \\
Publicar artículos científicos como profesional & $2,42 \pm 0,82$ \\
Participar en programas donde se aprenda y se realice investigación durante las vacaciones & $2,65 \pm 0,99$ \\
Participación y lectura de la revista científica & $2,66 \pm 1,02$ \\
Redactar el informe final de un estudio de investigación & $2,81 \pm 0,89$ \\
Realizar, publicar y presentar una investigación antes de graduarse & $2,98 \pm 1,25$
\end{tabular}




\begin{tabular}{lc}
\multicolumn{1}{c}{ Variable } & $\%$ \\
\hline Participación en los comités de facultad & $3,27 \pm 1,01$ \\
Realizar investigación científica durante la universidad & $3,27 \pm 1,03$ \\
Publicar artículos científicos durante la universidad & $2,92 \pm 1,41$ \\
$\begin{array}{l}\text { Presentar los trabajos de investigación en congresos y seminarios (charlas o pósteres) durante la } \\
\text { universidad }\end{array}$ & $3,11 \pm 1,22$ \\
$\begin{array}{l}\text { Presentar los trabajos de investigación en congresos y seminarios carrera universitaria (charlas o } \\
\text { pósteres) como profesional }\end{array}$ & $3,13 \pm 1,24$ \\
Otorgar créditos académicos a los estudiantes que realicen investigaciones, publicaciones y & $3,33 \pm 1,06$ \\
presentaciones científicas. & $3,18 \pm 1,27$ \\
Participación en el congreso y jornada científica de la facultad & $3,21 \pm 1,26$ \\
Realizar investigación científica como profesional &
\end{tabular}

*Datos expresados en promedio \pm desviación estándar.

Al comparar las carreras se encontró un mayor índice de disposición (Tabla 3) en estudiantes de enfermería, mientras que terapia ocupacional y fonoaudiología tuvieron la menor disposición frente a la investigación científica. De la muestra estudiada, la carrera que presentó estudiantes con una puntuación mayor o igual a 3,5 fue Enfermería $(33,3 \%)$, seguido por Kinesiología $(31,8 \%)$, mientras que Terapia Ocupacional mostró el porcentaje menor $(23,5 \%)$ en comparación con las demás carreras.

Tabla 3. Índice de disposición a la investigación y porcentaje de sujetos con mayor a 3,5, según de carrera

\begin{tabular}{lcccccc} 
& Nutrición & Kinesiología & Enfermería & $\begin{array}{c}\text { Terapia } \\
\text { Ocupacional }\end{array}$ & Fonoaudiología & P \\
& & & & & \\
\hline Índice de disposición* & $2,71 \pm 1,01^{\mathrm{ab}}$ & $2,8 \pm 1,05^{\mathrm{a}}$ & $2,83 \pm 1,16^{\mathrm{a}}$ & $2,49 \pm 0,93^{\mathrm{b}}$ & $2,49 \pm 0,99^{\mathrm{b}}$ & 0,02 \\
Sujetos con puntuación $\geq 3,5 \dagger$ & $29,4 \%$ & $31,8 \%$ & $33,3 \%$ & $23,5 \%$ & $26,3 \%$ & 0,03
\end{tabular}

* Datos expresados en promedio \pm desviación estándar. Prueba de ANOVA con Post-Hoc Tukey. Nivel de significancia p=,05

ab Letras diferentes en misma fila indican diferencias significativas.

$\dagger$ Datos expresados en porcentajes. Test de Chi2, nivel de significancia $p=, 05$.

\section{DISCUSIÓN}

Esta investigación tuvo como propósito evaluar la disposición que tienen los alumnos de ciencias de la salud hacia la investigación científica. Nuestros resultados muestran que la disposición que tienen los estudiantes con relación a la investigación es baja, por debajo del punto de corte asumido como aceptable para esta investigación.

Nuestros hallazgos son similares a los presentados por Silva et al. (2013) en estudiantes de Panamá donde también se encontró un nivel regular de actitud frente a la investigación la cual decrece a medida que avanzan en el plan de estudio. Los investigadores reportaron valores promedios de $2,98 \pm 0,59$ lo que resulta un poco mayor a lo reportado en este estudio ${ }^{(4)}$.

Los valores más bajos se encontraron en ítems relacionados con las búsquedas bibliográficas y a la dificultad en la utilización de programas estadísticos. En este sentido nuestros resultados difieren a lo reportado por Peña et al. (2015), en su estudio con estudiantes cubanos donde se reporta una actitud positiva hacia la estadística (6). En general, las profesiones de salud tienen un enfoque disciplinario particular que hace que las asignaturas y operaciones que requieren de la lógica matemática sean las menos preferidas ${ }^{(7,8)}$.

Por otro lado, nuestros hallazgos revelan que los estudiantes con una actitud más positiva respecto a la investigación son los que estudian enfermería. Resultados similares fueron reportados por Ochoa et al. (2016) en su estudio con estudiantes peruanos de enfermería dónde más del 65 \% presentó una actitud favorable a la investigación, lo que concuerda con los presentado por Trujillo et al. (2015), en estudiantes mexicanos de enfermería ${ }^{(9,10)}$. 
En conclusión, nuestros resultados muestran una actitud entre regular a negativa hacia a la investigación por parte de estudiantes universitarios de ciencias de la salud, siendo los estudiantes de Terapia Ocupacional y Fonoaudiología los que reportan valores más bajos. Por tanto, es necesario fomentar en el estudiantado el reconocimiento de la importancia de basamento científico en el abordaje de situaciones de salud y a partir de este formular posibles mecanismos de intervención con un carácter ético.

Se recomienda futuras investigaciones con mayor tamaño muestral e incorporar a los alumnos que se encuentran en prácticas profesionales, así como utilizar técnicas cualitativas que permitan describir las razones que subyacen en estas actitudes negativas hacia la investigación.

\section{REFERENCIAS BIBLIOGRÁFICAS}

1. Plazas Vargas Merideidy, Gómez Suarez Marcela, Castro Moreno Carlos Alberto. Actitud en estudiantes de Ciencias de la Salud hacia el conocimiento científico. Rev Cienc. Salud. 2013; 11(1):83-91. Disponible en: http://www.scielo.org.co/ $\mathrm{pdf} /$ recis/v11n1/v11n1a06.pdf

2. Álvarez-Muñoz P. Pérez-Montoro M. Políticas científicas públicas en Latinoamérica. Prof Inf. 2016; 25(5):758-766. Disponible en: https://recyt.fecyt.es/index.php/EPI/article/ view/epi.2016.sep.06

3. Sánchez-Duque J. Gómez-González J, Rodríguez-Morales A. Publicación desde el pregrado en Latinoamérica: dificultades y factores asociados en estudiantes de Medicina. Investigación educ médica. 2017; 22 (6):104-108. Disponible en: https://www.sciencedirect.com/science/article/pii/ S2007505716300424

4. Silva $S$, Zúñiga-Cisneros J, Ortga-Loubon Ch, Yau A, Castro $\mathrm{F}$, Barría-Castro J, et al. Conocimientos y actitudes acerca de la investigación científica en los estudiantes de medicina de la Universidad de Panamá. Arch Med. 2013; 9(3): 1-10. Disponible en: http://www.archivosdemedicina.com/ medicina-de-familia/conocimientosy-actitudes-acercadela-investigacincientfica-en-losestudiantes-demedicina-delauniversidad-depanam. php?aid $=613$

5. Pelcastre Villafuerte L, Gómez Serrato AR, Zavala G. Actitudes hacia la ciencia de estudiantes de educación preuniversitaria del centro de México. Rev Eurek. 2015; 12(3):475-490. Disponible en: http://rodin.uca.es/xmlui/ handle/10498/17603

6. Peña Rodríguez A, Suárez Martínez R, Sanjuán Gomez G, Rabell Piera O, Gómez Martínez M, Morales Velázquez IC. Actitudes hacia la asignatura de Estadística en estudiantes de la Facultad de Ciencias Médicas “General Calixto García”. Rev
Haban Cienc Méd. 2015; 14(6): 872-883. Disponible en: http: / / scielo.sld.cu/scielo. php?script=sci_arttext\&pid=S1729519X2015000600016

7. Torres Aviles F, Aparicio Pereda A, Bazán Guzman J, Joao Abdounur 0. Actitudes hacia la estadística en Universitarios del Área de las ciencias de una universidad pública. Educ Matem Pesq Sao Paulo. 2015; 17(1):45-73. Disponible en: http://www.producao.usp.br/bitstream/handle/ $\mathrm{BDPI} / 51337 / 2713161$. pdf?sequence=2\&isAllowed $=\mathrm{y}$

8. Rodríguez Feijóo N. Actitudes de los estudiantes universitarios hacia la Estadística. Interdisciplinaria. 2011; 28(2):199205. Disponible en: http://www.scielo.org.ar/scielo. php?script=sci_arttext\&pid=S1668-70272011000200002

9. Ochoa-Vigo K, Bello Vidal C, Villanueva Benites M, Ruiz-Garay M, Manrique Borjas G. Percepción y actitud del universitario de enfermería sobre su formación en investigación. Rev Med Hered. 2016; 27(4):204-215. Disponible en: http://www. scielo.org.pe/scielo.php?script=sci_arttext\&pid=S1018$130 \times 2016000400003$

10. Alonso Trujillo J, Alonso Ricardez A, Valadez Diaz D. Actitud hacia la investigación científica de estudiantes de enfermería. Cuid Arte "el arte del cuidado", 2015; 4(7): 24-35. Disponible en: http://journals.iztacala.unam.mx/index.php/cuidarte/ article/view/329

Fuentes de financiamiento:

Este artículo ha sido financiado por los autores.

Conflictos de interés:

Los autores declaran no tener ningún conflicto de interés.

Correspondencia:

Eduard Maury-Sintjago

Dirección: Av. Andrés Bello $N^{\circ}$ 720. Chillán, Chile.

Teléfono: +56422463294

Correo electrónico: emaury@ubiobio.cl

Recibido: 30 de enero de 2018

Evaluado: 01 de febrero de 2018

Aprobado: 20 de marzo de 2018

(c) La revista. Publicado por Universidad de San Martín de Porres, Perú. (cc) $\mathbf{B r}$ Licencia de Creative Commons Artículo en acceso abierto bajo términos de Licencia Creative Commons Atribución 4.0 Internacional. (http://creativecommons.org/licenses/by/4.0/)

\section{ORCID iDs}

Eduard Antonio Maury-Sintjago Estefanía Valenzuela-Figueroa Massiel Henríquez-Riquelme Alejandra Rodríguez-Fernández

https: / / orcid.org/0000-0002-9482-8268 https://orcid.org/0000-0002-3949-8358 https://orcid.org/0000-0002-8677-6429 https: / / orcid.org/0000-0002-3982-3165 